\title{
Role of a Dentist in the Diagnosis and Treatment of Obstructive Sleep Apnea
}

\author{
${ }^{1}$ V Naveen Kumar, ${ }^{2}$ Nourah A Kader, ${ }^{3}$ Govindarajan Sujatha, ${ }^{4}$ Manikandan Gunasekaran, ${ }^{5}$ Shankargouda Patil, \\ ${ }^{6}$ A Thirumal Raj
}

How to cite this article: Kumar VN, Kader NA, Sujatha G, Gunasekaran M, Patil S, Raj AT. Role of a Dentist in the Diagnosis and Treatment of Obstructive Sleep Apnea. World J Dent 2018;9(6)437-438.

\section{Source of support: Nil}

Conflict of interest: None

Obstructive sleep apnea (OSA) is a chronic progressive disease involving recurring episodes of partial/total obstruction of the upper airway during sleep. ${ }^{1}$ Prevalent amidst $2-4 \%$ of middle-aged individuals, the disorder finds a higher incidence rate among men (10-20\%). ${ }^{1,2}$ This condition may be associated with cardiovascular disorders (CVD), hypertension, type II diabetes, attention-deficit hyperactivity disorder (ADHD), immune system deficiencies, and accidents. ${ }^{1}$ A study by Dillow et al. ${ }^{3}$ showed that a significant number of patients sought physician consultations after receiving a warning from dentists of potential diagnosis of OSA. Dentist in conjunction with a sleep medicine physician are well equipped to arrive at a definitive diagnosis for this condition. Noticeable predictors to a dentist occur in the form of nasal septum deviation, sinusitis, macroglossia, macrosomia, and even obesity. A welcome relief and a more actively

${ }^{1}$ Department of Conservative Dentistry and Endodontics, Karpaga Vinayaga Institute of Dental Sciences, Kanchipuram, Tamil Nadu, India.

${ }^{2}$ All Smiles Dental Clinic, Chennai, Tamil Nadu, India.

${ }^{3}$ Department of Oral Pathology and Microbiology, Sri Venkateswara Dental College and Hospital, Chennai, Tamil Nadu, India

${ }^{4}$ Department of Dentistry, Shri Sathyasai Medical College and Research Institute, Sri Balaji Vidyapeeth University, Nellikuppam, Tamil Nadu, India

${ }^{5}$ Department of Maxillofacial Surgery and Diagnostic Sciences, Division of Oral Pathology, College of Dentistry, Jazan University, Jazan, Kingdom of Saudi Arabia

${ }^{6}$ Department of Oral Pathology and Microbiology, Sri Venkateswara Dental College and Hospital, Chennai, Tamil Nadu, India

Corresponding Author: A Thirumal Raj, Department of Oral Pathology and Microbiology, Sri Venkateswara Dental College and Hospital, Chennai, Tamil Nadu, India, e-mail: thirumalraj666@gmail.com practiced technique among dentists is the incorporation of oral appliances as a form of treatment. This can positively affect prognosis and improve quality of life for the patient. Oral appliances reduce the nasal airway obstruction, by modulating the mandible to a protruded position. Success rates have been satisfactory for both mild and severe cases. ${ }^{1}$ Oral protrusion devices (with or without expansion screw), tongue retainers, rapid maxillary expansion, and mandibular advancement splints are alternatively employed on a case-to-case basis. Though successful, oral appliances demand caution. The dentist performing this treatment must be well-versed with the polysomnographic examination, pulse oximetry, oral appliance mechanics and customizing insight based on the patient's physiology and systemic complications. If administered inappropriately, this method could lead to complication such as skeletal and occlusal changes, temporomandibular joint (TMJ) deformities, musculoskeletal disorders, pain, sialorrhea, etc. ${ }^{1,3}$

Preventive care is pivotal in OSA. It also greatly reduces the risk of subsequent systemic complications. Promoting awareness around the subject, its causes, symptoms, and consequences are vital. In many instances, OSA is left untreated as they are often misdiagnosed as depression, anxiety, poor cognitive ability and other systemic conditions like CVD and hypertension (which are in fact are the consequences of OSA). OSA requires a multidisciplinary approach from diagnosis to treatment planning. The dentist must be a part of an interdisciplinary model involving physicians from sleep medicine, psychiatry, pulmonology, ear, nose, throat (ENT), and others to aid in early diagnosis and to collectively improve the patient's quality of life. Oral appliances, if deemed necessary must be administered with utmost care and proactively followed up. Radiographs must be recorded before treatment, with other records, such as polysomnography, electrocardiogram (ECG), electrocenphalogram (EEG), pulse oximetry and study models of existing occlusions recorded during its course. Monitoring improvements continuously and tracking records could limit the damages to skeletal and muscular elements caused by devices that protrude the mandible. While we have sufficient literature to showcase the satisfactory treatment outcomes on OSA (in its mild and severe forms) with oral appliances, we must invest in further research to limit its disadvantages. 


\section{REFERENCES}

1. Range AJA, Barros VM, Seraidarian PI. Snoring and Obstructive Sleep Apnea Syndrome: A reflection on the role of Dentistry in the current scientific scenario. Dental Press J Orthod. 2012;17(3):58-63
2. Olson LG, King MT, Hensley MJ, Saunders NA. A community study of snoring and sleep-disordered breathing. Prevalence. Am J Respir Crit Care Med. 1995;152(2):711-716.

3. Dillow K, Gregory, Sanders A, Sheats R, Brame J. Patient response to sleep apnea screening in a dental Practice; J Public Health Dent. 2017;77:13-20. 